\title{
Serum Exosomal EphA2 is a Prognostic Biomarker in Patients with Pancreatic Cancer
}

\author{
Qian Wei \\ Ze $\mathrm{Li}$ (D) \\ Honglei Feng \\ Li Ren
}

Department of Laboratory, Tianjin Medical University Cancer Institute and Hospital, National Clinical Research Center for Cancer, Key Laboratory of Cancer Prevention and Therapy, Tianjin's Clinical Research Center for Cancer, Tianjin, 300060, People's Republic of China
Correspondence: Li Ren

Department of Laboratory, Tianjin

Medical University Cancer Institute and

Hospital, West Huan-Hu Road, Ti Yuan

Bei, Hexi District, Tianjin, 300060,

People's Republic of China

Email lirentj@I63.com
Background: Pancreatic cancer (PC) is one of the worst prognoses amongst all malignant diseases. It is therefore of great significance to identify biomarkers with predictive clinical value for the prognosis and recurrence of PC.

Methods: In our study, enzyme-linked immunosorbent assays (ELISA) were used to detect the expression of Exo-EphA2 in the serum of PC patients and controls. Kaplan-Meier curve and Cox regression analyses were used to evaluate the prognostic value of Exo-EphA2 expression in patients with primary and recurrent PC.

Results: The level of serum Exo-EphA2 was significantly higher in the PC group when compared to that of the control group. High expression of Exo-EphA2 in PC was associated with shorter overall survival (OS) and proved to be a significant negative prognostic factor in the multivariate analysis ( $\mathrm{HR}=1.04,95 \% \mathrm{CI}: 1.00-1.09, \mathrm{P}<0.001)$. Additionally, we found that the level of serum Exo-EphA2 in recurrent PC patients (first recurrence $<12$ months) was positively correlated with the level of Exo-EphA2 at primary diagnosis. Multivariate analysis showed that a high expression of Exo-EphA2 in recurrent PC was associated with shorter recurrence-free survival (RFS) (HR $=1.41,95 \% \mathrm{CI}: 1.10-1.70, \mathrm{P}<0.001$ ).

Conclusion: High expression of serum Exo-EphA2 represents a novel biomarker for a poor prognosis in PC patients.

Keywords: pancreatic cancer, exosome, EphA2, prognosis

\section{Introduction}

Pancreatic cancer (PC) is one of the most lethal malignancies with a five-year survival rate of approximately $5 \%$, and is the fourth leading cause of cancer-related deaths globally. ${ }^{1,2}$ Because of its invasiveness and high metastasis rate, surgical resection remains the most effective treatment with prolonged median survival of 12.6 months. $^{3}$ However, it remains difficult to predict the prognosis based on resection alone. Therefore, there is a high unmet medical need to identify molecular biomarkers for the prognosis, and develop individualized therapy to improve the survival of PC patients.

Accumulating evidences have shown that exosome signaling pathways are involved in cancer progression. ${ }^{4}$ Exosomes are small membranes vesicles (40$150 \mathrm{~nm})$ containing various active biological molecules involved in intercellular communication and tumor progression. ${ }^{5}$ Cancer cells can translocate growth factors and cytokines to the surface and release exosomes to communicate with other cells in the environment, leading to establish the invasive growth of a tumor. ${ }^{6}$ For example, recent research indicates that exosomes from pancreatic ductal adenocarcinoma cells induce mouse liver Kupffer cells to secrete transforming factor $\beta$ 
(TGF- $\beta$ ) and to upregulate the expression of fibronectin in hepatic stellate cells. ${ }^{7}$ In addition, with the rise of liquid biopsies, circulating exosomes have been widely reported as noninvasive biomarkers for the diagnosis and prognosis of various malignancies, such as liver, colorectal, and prostate cancer. ${ }^{8-10}$ For example, serum exosomal miR$125 \mathrm{~b}$ was reported as a novel prognostic marker for hepatocellular carcinoma with high specificity and sensitivity. Serum exosomal miR-122 has been considered a potential biomarker for diagnosis and prognosis of colorectal cancer with liver metastasis. Similarly, plasma exosomal miR-141 and miR-375 were overexpressed in patients with recurrent prostate cancer after radical prostatectomy compared to patients with non-recurrent disease.

Ephrin type-A receptor 2 (EphA2) is overexpressed in multiple types of cancers such as breast, melanoma, ovarian adenocarcinoma. ${ }^{11-13}$ Due to its strong association with tumor progression, metastasis, and prognosis, EphA2 is considered a possible therapeutic target, as well as a diagnostic and prognostic marker. A previous study indicated that EphA2-enriched exosomes (ExoEphA2) could induce chemoresistance in PC cells, which could help predict the biochemical response to gemcitabine-based chemotherapy. ${ }^{14}$ Additionally, Exo-EphA2 acts as an oncogene in PC. In support of these findings, we observed that Exo-EphA2 could transfer metastatic potential to recipient cells. ${ }^{15}$ Consistent with this, the level of circulating Exo-EphA2 was higher in PC patients when compared to that of healthy controls, suggesting it could serve as a potential diagnostic marker for PC. ${ }^{16}$ However, the relationship between the underlying dynamic expression of serum Exo-EphA2 and the prognosis and recurrence of $\mathrm{PC}$ remains unclear.

Therefore, we evaluated the predictive value of serum Exo-EphA2 levels for prognosis and recurrence of PC.

\section{Patients and Methods}

\section{Patients and Samples}

We collected 200 serum samples from patients with PC, benign pancreatic disease and healthy controls at the Tianjin Medical University Cancer Institute and Hospital (China) from January 2017 to December 2017. After centrifugation at $3000 \mathrm{rpm}$ for $10 \mathrm{~min}, 1 \mathrm{~mL}$ of serum samples collected from venous blood were transferred to fresh tubes. All patients received a regular telephone follow-up every three months to record 1-year recurrence rates and 3-year survival rates. The tumor stage was classified according to the American Joint Committee on Cancer (AJCC) TNM classification. Clinical samples were collected from all participants after obtaining informed consents.

\section{Exosome Isolation and Purification}

We used the ExoQuick Exosome Precipitation Solution (System Biosciences, Mountain View, USA) to precipitate exosomes from $250 \mu \mathrm{L}$ of serum, according to the manufacturer's protocol. In short, $250 \mu \mathrm{L}$ of serum and $63 \mu \mathrm{L}$ of EXO-Quick solution were mixed and refrigerated at $4{ }^{\circ} \mathrm{C}$ for $30 \mathrm{~min}$. Then, we centrifuged the mixture at $1,500 \mathrm{xg}$ at $4{ }^{\circ} \mathrm{C}$ for $30 \mathrm{~min}$. The pelleted exosomes were suspended in $100 \mu \mathrm{L}$ of PBS solution. After that, the mixture was centrifuged at $100,000 \mathrm{xg}$ for $60 \mathrm{~min}$ to purify exosomes. The pelleted exosomes were resuspended in $100 \mu \mathrm{L}$ of PBS solution and frozen at $-80{ }^{\circ} \mathrm{C}$ until further processing. Unnecessary freeze-thaw cycles were avoided.

\section{Exosome Quantification}

Quantification of serum exosomes was performed using the ExoQuant ${ }^{\mathrm{TM}}$ Overall Exosome Capture and Quantification Assay Kit (ELISA). (Biovision, CA, USA) according to the manufacturer's protocols. Briefly, $100 \mu \mathrm{L}$ of serum samples were added to 96-well plates and shaken at room temperature for $30 \mathrm{~min}$. The plate was then incubated at $4^{\circ} \mathrm{C}$ overnight. Wash the plates three times with $300 \mu \mathrm{L} /$ well of Washing Buffer. Then $100 \mu \mathrm{L}$ of exosome detection antibody (1:500) was added to each well and incubate at room temperature for $15 \mathrm{~min}$ and at $4^{\circ} \mathrm{C}$ for $2 \mathrm{~h}$. After washing the plate three times, $100 \mu \mathrm{L}$ of Streptavidin-HRP antibody solution was added to each well and incubate at room temperature while shaking for $15 \mathrm{~min}$. This solution was then kept at $4^{\circ} \mathrm{C}$ for $1 \mathrm{~h}$. Next, $100 \mu \mathrm{L}$ of substrate chromogenic solution was added to each well and incubate at room temperature in the dark for 5-10 min. The reaction was stopped by the addition of 100 $\mu \mathrm{L}$ of stopping solution and the absorbance recorded at $450 \mathrm{~nm}$ within 10 minutes.

\section{Transmission Electron Microscopy Analysis}

The exosomes were examined by transmission electron microscopy (TEM) as described previously. ${ }^{15}$ Briefly, purified exosomes was loaded onto non-glow-discharged carbon-coated grids for $10 \mathrm{~min}$ with $4 \%$ paraformaldehyde and negatively stained with $2 \%$ uranyl acetate for $1 \mathrm{~min}$ at 
room temperature. The fried grids were viewed under a FEI/Philips CM12 TEM operated at $80 \mathrm{kV}$.

\section{Western Blot Analysis}

Proteins were extracted from the exosome pellets using RIPA buffer (Thermo Fisher Scientific, Waltham, USA). The protein concentration was determined using a BCA kit (Thermo Fisher Scientific, Waltham, USA). An amount of exosome lysate $(10 \mu \mathrm{g})$ was run on $10 \%$ SDS-PAGE (Bio-Rad Laboratories, Hercules, USA), transferred to nitrocellulose membranes and blocked in PBS with $0.05 \%$ Tween-20 (PBST) containing 5\% skimmed milk at room temperature for $2 \mathrm{~h}$. The membranes were incubated with anti-CD63 (Abcam; 1:500), anti-CD9 (Abcam; 1:500) or GAPDH at $4^{\circ} \mathrm{C}$ overnight. The membranes were washed four times with PBST and incubated with an appropriate secondary antibody for $1 \mathrm{~h}$ at room temperature. Protein bands were visualized using ECL Western Blotting Detection Reagent (ABM Inc., Canada) and analyzed with ImageJ software (version 1.8.0).

\section{Detection of Exo-EphA2}

Concentrations of serum Exo-EphA2 levels were determined using the enzyme-linked immunosorbent assay (ELISA) kits as described by Wei et al ${ }^{16}$ (Raybiotech, Atlanta, USA). Details of the procedure are outlined in the manufacturer's instructions. In short, a 96-well microplate was coated with $100 \mu \mathrm{L}$ of anti-human EphA2 antibody overnight before use. Plates were blocked at room temperature for 1 hour, after which serum samples were added and incubated at room temperature for 2.5 hours. After washing three times, the samples were incubated with the detection antibody for another two hours at room temperature, followed by the addition of a streptavidin solution for 45 minutes. Finally, the plates were washed three times, a chromogenic was added for 30 minutes, and finally the reaction was stopped using a stop solution. A microplate reader (Model 680, Bio-Rad Laboratories, Hercules, USA) was used to measure the absorbance at $450 \mathrm{~nm}$. All samples were measured in duplicate.

\section{Statistical Analysis}

The statistical analyses were conducted with the Statistical Package for Social Scientists (SPSS, version 23.0; IBM Corp, Armonk, USA) and GraphPad Prism Software 5.0 (GraphPad Software Inc, San Diego, USA). All data were expressed as median and interquartile range. The Wilcoxon
Signed-Rank test was used to compare levels of serum ExoEphA2 between groups. Differences in overall survival (OS) and recurrence-free survival (RFS) in different subgroups were visualized using Kaplan-Meier plots and differences between groups were analyzed using the Log rank test. Univariate and multivariate Cox regression analyses were performed to study the prognostic relevance of Exo-EphA2 in PC. The correlation between serum Exo-EphA2 at primary diagnosis and recurrence was assessed using Spearman's rank-order analysis. $P<0.050$ was considered statistically significant. All experiments were performed three times.

\section{Results \\ Clinical Characteristics of the Patient Cohort}

We analyzed 200 serum samples, including 90 serum samples of PC patients before any therapeutic procedure (chemotherapy and/or chemoradiotherapy), 55 patients with a benign pancreatic disease, and 55 age-matched healthy controls. The 90 PC patients consisted of a male/ female ratio of 2:1 and a median age of 60 years (ranging from 28 to 87 years). According to the AJCC staging TNM classification, patients were divided into those with early-stage (stage I/II) and those with advanced-stage (stage III/IV). Furthermore, all 90 patients were treated with gemcitabine-based chemotherapy after surgery. Detailed data on clinical parameters, including age, gender, drinking and smoking habits, hypertension, lymph node metastasis, and stage, are summarized in Table 1.

\section{Validation of the Isolated Serum Exosomes}

Isolated serum exosomes were identified by TEM and Western blot. As shown in Figure 1A, the exosome structure and shape were observed via TEM. Western blot showed that the exosomal markers CD9 and CD63 were both enriched in the purified exosome pellets (Figure 1B). These results indicated that the isolated exosomes were purified adequately. Furthermore, we observed that the relative expression of exosomes in the serum of $\mathrm{PC}$ patients did not differ from that of benign pancreatic disease patients or healthy controls (Figure 1C).

\section{Serum Exo-EphA2 is Elevated in Pancreatic Cancer}

We detected the expression of Exo-EphA2 in the 200 serum samples. As shown in Figure 2A, serum Exo-EphA2 levels 
Table I Clinic Characteristics of Pancreatic Cancer Patients

\begin{tabular}{|c|c|}
\hline Characteristics & $(\mathrm{N}=90)(\%)$ \\
\hline \multicolumn{2}{|l|}{ Age } \\
\hline$<55$ & $30(33.3)$ \\
\hline$>55$ & $60(66.7)$ \\
\hline \multicolumn{2}{|l|}{ Gender } \\
\hline Female & $29(32.2)$ \\
\hline Male & $61(67.8)$ \\
\hline \multicolumn{2}{|l|}{ Smoke } \\
\hline Yes & $35(38.9)$ \\
\hline No & $55(6 I .1)$ \\
\hline \multicolumn{2}{|l|}{ Drink } \\
\hline Yes & $2 I(23.3)$ \\
\hline No & $69(76.7)$ \\
\hline \multicolumn{2}{|l|}{ Hypertension } \\
\hline Yes & $20(66.7)$ \\
\hline No & $70(66.7)$ \\
\hline \multicolumn{2}{|c|}{ Lymph nodes metastasis } \\
\hline Yes & $58(64.4)$ \\
\hline No & $32(35.6)$ \\
\hline \multicolumn{2}{|l|}{ TNM stage } \\
\hline Stage I/II & $60(66.7)$ \\
\hline Stage III/IV & $30(33.3)$ \\
\hline \multicolumn{2}{|c|}{ Recurrence (I year) } \\
\hline Yes & $5 \mathrm{I}(56.7)$ \\
\hline No & $39(43.3)$ \\
\hline \multicolumn{2}{|l|}{ Overall survival } \\
\hline Survival & $7(7.8)$ \\
\hline Death & $83(92.2)$ \\
\hline
\end{tabular}

in PC patients were significantly higher than those of benign pancreatic disease patients and healthy controls. In addition, serum Exo-EphA2 levels in PC patients with advanced-stage (III+IV) were significantly higher than those of PC patients with early-stage (I+II) (Figure 2B).

\section{Serum Exo-EphA2 Level is an Independent Predictor for OS in Pancreatic Cancer}

To investigate the prognostic value of Exo-EphA2 in PC, we analyzed the 3-year overall survival of patients with early and advanced disease stages. The 90 PC patients were divided into two groups based on the median level of Exo-EphA2 expression. Kaplan-Meier curves of OS showed that a higher Exo-EphA2 expression was significantly associated with a poorer outcome. The median OS time was 17 months in the high Exo-EphA2 group and 31 months in the low Exo-EphA2 group (Figure 3A). Subgroup analysis showed that high Exo-EphA2 significantly affected OS in patients with early $(P<0.001)$ and advanced stage cancer $(P=0.036)$ (Figure $3 \mathrm{~B}$ and $\mathrm{C}$ ). Univariate analysis suggested that positive lymph nodes $(P=0.03)$, tumor stage $(P=0.01)$, and Exo-EphA2 expression $(P=0.02)$ were associated with a poor OS, but not age $(P=0.39)$, gender $(P=0.76)$, drinking $(P=$ $0.63)$, smoking $(P=0.19)$ or hypertension $(P=0.13)$. Multivariate analysis revealed that Exo-EphA2 is indeed an independent risk factor for OS in PC patients (HR = 1.04, 95\% CI: 1.00-1.09, $P<0.001$; Table 2).

\section{Serum Exo-EphA2 Levels in Recurrent Pancreatic Cancer}

Among 90 PC patients, 51 patients experienced recurrence within one year. From those 51 patients, the corresponding serum samples at primary diagnosis and recurrence were available. The Exo-EphA2 levels in patients with recurrent PC were significantly higher than those of patients with non-recurrent PC (Figure 4A). Moreover, we found a strong correlation between the Exo-EphA2 level at the primary diagnosis and at the first recurrence $(\mathrm{R}=0.668$, $P<0.001$; Figure 4B).

\section{Serum Exo-EphA2 Level is an Independent Predictor of RFS in Pancreatic Cancer}

A high expression of Exo-EphA2 was significantly associated with poor RFS (Figure 5A). Subgroup analysis identified that high Exo-EphA2 expression was significantly associated with RFS in patients with early stage $(\mathrm{P}<0.001)$, but not in patients with advanced stage cancer $(\mathrm{P}=0.105)$ (Figure 5B and $\mathrm{C})$. Univariate analysis showed that positive lymph nodes $(\mathrm{P}=0.03)$, tumor stage $(\mathrm{P}=$ $0.01)$ and Exo-EphA2 expression $(\mathrm{P}=0.01)$ were associated with RFS, but not age $(\mathrm{P}=0.53)$, gender $(\mathrm{P}=0.34)$, drinking $(\mathrm{P}=0.83)$, smoking $(\mathrm{P}=0.53)$ or hypertension $(\mathrm{P}$ $=0.72$ ). The multivariate analysis confirmed that ExoEphA2 was an independent risk factor for RFS in PC patients. $(\mathrm{HR}=1.41,95 \% \mathrm{CI}: 1.10-1.70, \mathrm{P}=0.001$; Table 3). 
A

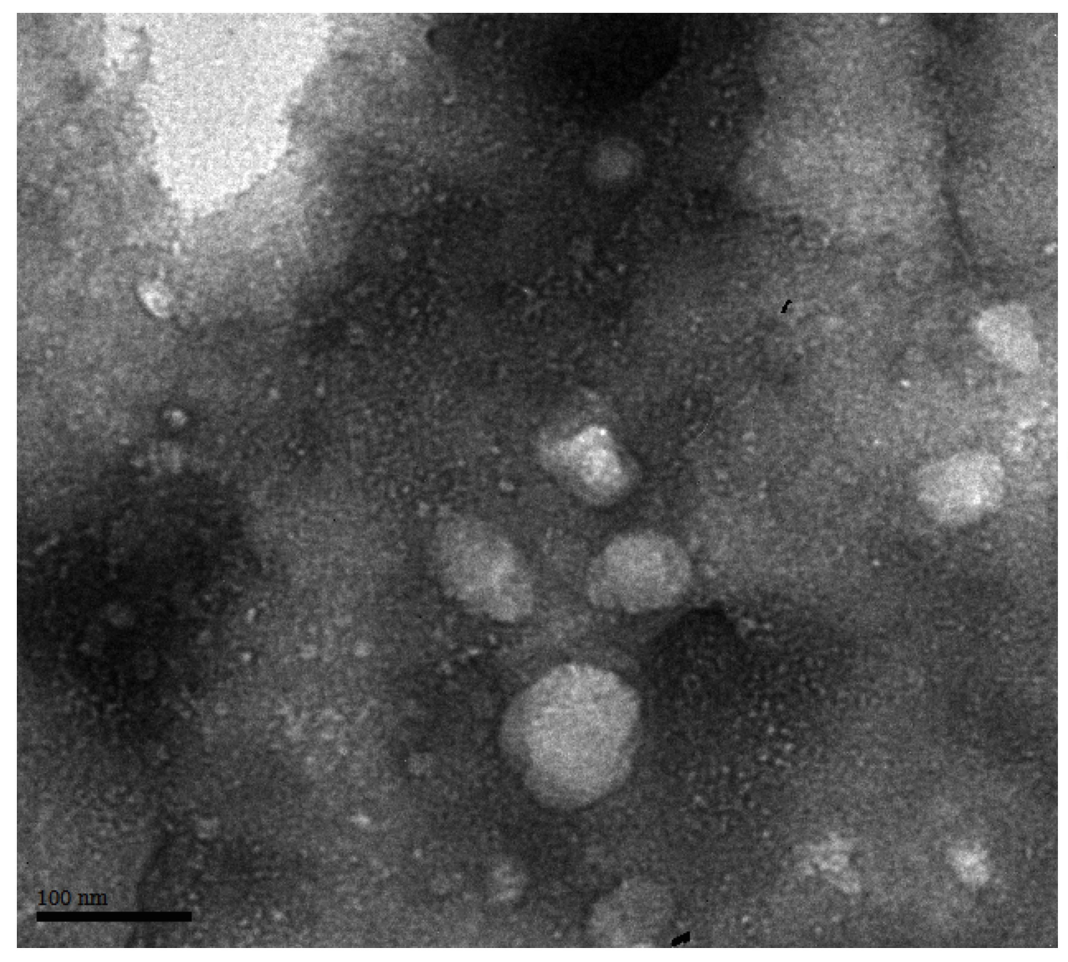

B

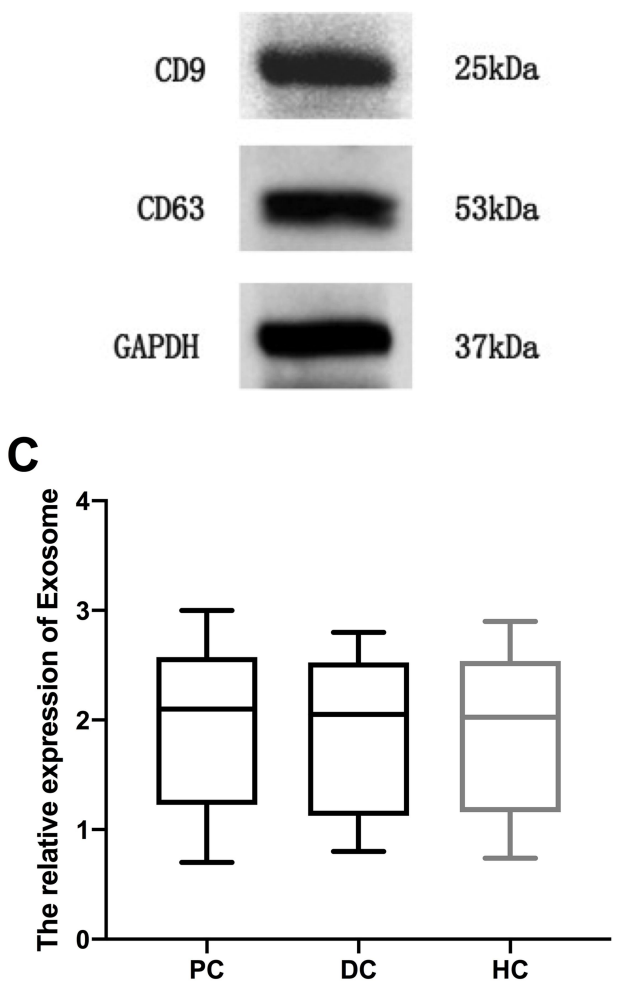

Figure I The validation of isolated serum exosomes. (A) Transmission electron microscopy micrographs showing exosome morphology and size. (B) Western blotting showing the expression of exosomal positive marker CD9 and CD63. (C) Exosome capture and quantification assay showing the relative expression of exosome in different cohort.

Abbreviations: PC, pancreatic cancer; DC, benign disease; HC, healthy control.

A

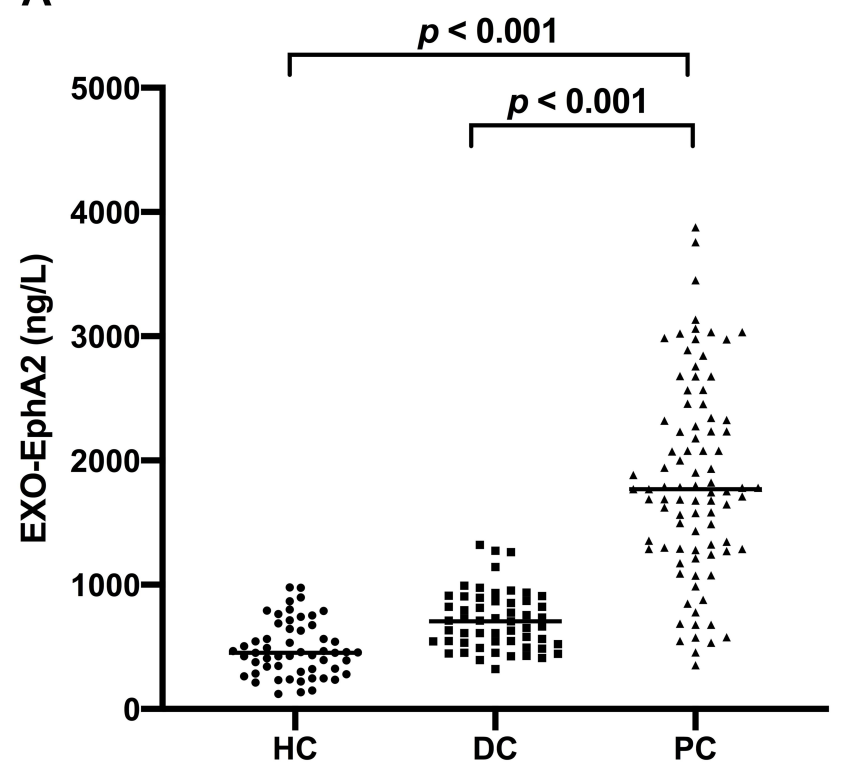

B

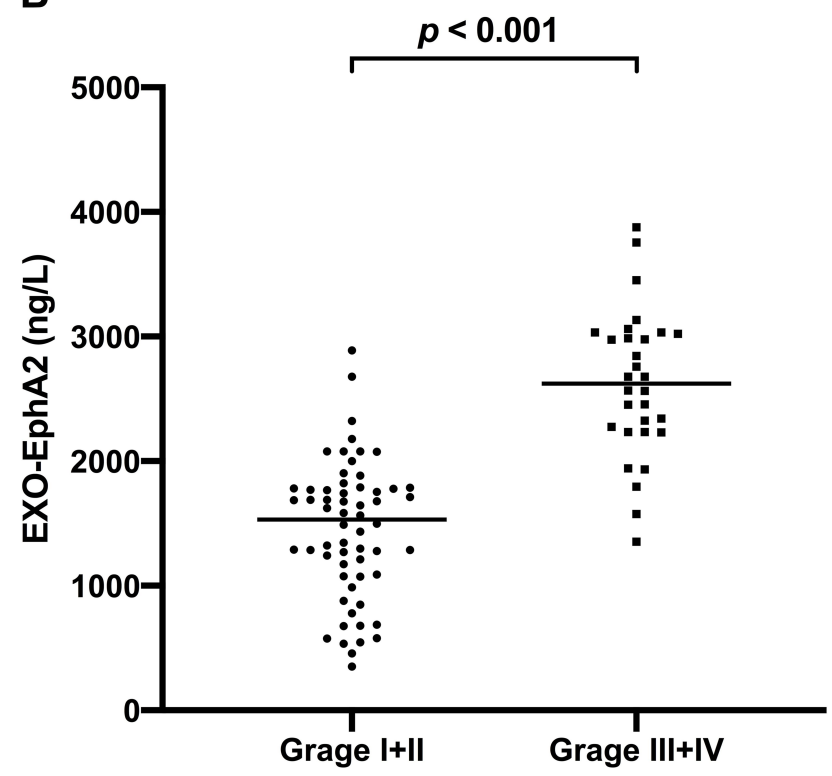

Figure 2 Serum Exo-EphA2 is upregulated in pancreatic cancer. (A) Expression of serum Exo-EphA2 in pancreatic cancer, benign pancreatic disease and healthy controls $(p<0.00 \mathrm{I})$. (B) Expression of serum Exo-EphA2 in PC Stage I/II and PC Stage III/IV $(p<0.00 \mathrm{I})$.

Abbreviations: PC, pancreatic cancer; DC, benign disease; HC, healthy control. 

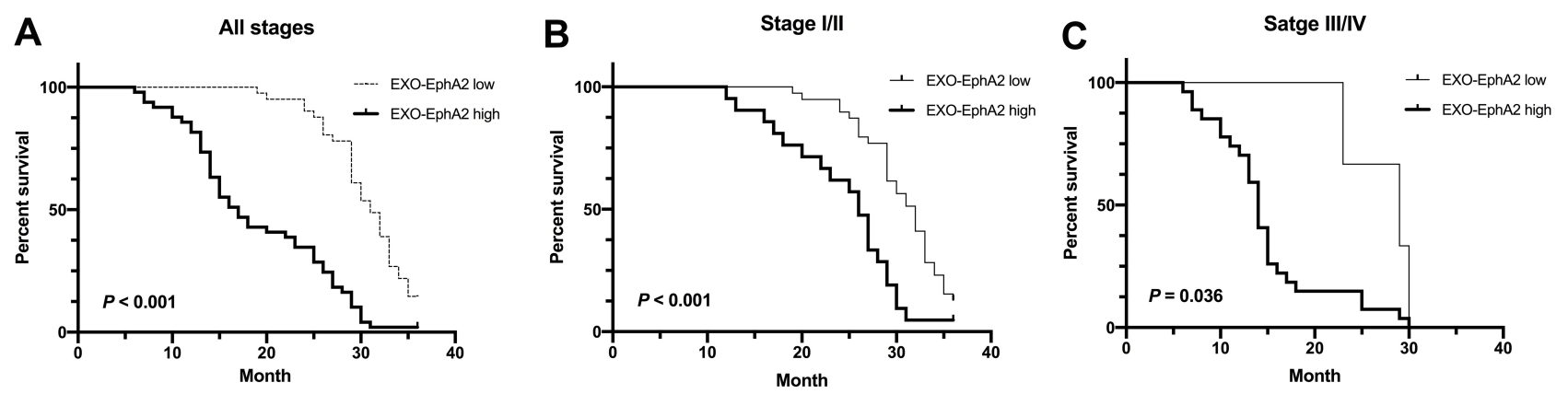

Figure 3 Higher Exo-EphA2 expression was significantly associated with poor overall survival (OS). Kaplan-Meier curves of OS (A) in pancreatic cancer ( $p<0.00 \mathrm{I})$, (B) in stage I/II $(p<0.00 \mathrm{I})$ and $(\mathbf{C})$ in stage III/IV $(p=0.036)$. Based on the median level of Exo-EphA2 expression (I768 ng/L), pancreatic cancer patients were divided into two groups (EphA2-high expression and EphA2-low expression).

\section{Discussion}

There is a high incidence of postoperative recurrences in PC patients due to histological aggressiveness and chemotherapy tolerance of the malignant tumor. ${ }^{17}$ Therefore, early diagnosis and surgical therapy are the preferred treatment. However, in clinical practice, the effect of only surgical treatment alone is limited, as well as radiotherapy, chemotherapy, and targeted therapy. ${ }^{18,19}$ Despite recent progress in diagnosing and treating PC, the prognosis remains poor due to frequent postoperative recurrences and metastasis. ${ }^{20}$ Therefore, to improve prognosis, the identification of novel prognostic biomarkers and specific signaling pathways are vital to stratify the risk of recurrence and identify new potential therapeutic targets.

Exosomes mediate the interaction between cells and their surrounding microenvironment by carrying bioactive molecules. $^{21-23}$ In recent years, more attention has been paid to exosome signaling pathways as diagnostic and prognostic biomarkers for multiple solid tumors. For example, circulating exosomal miR-150-5p acted as a novel diagnostic and prognostic marker for colorectal cancer, which in combination with carcinoembryonic antigen (CEA), could improve the diagnostic accuracy. ${ }^{24} \mathrm{An}$ increased expression of exosomal miR-17-5p in breast cancer suggested that it might serve as a potential diagnostic biomarker for breast cancer with $66.67 \%$ sensitivity and $83.59 \%$ specificity. ${ }^{25}$ In addition to miRNAs, exosome proteins also play essential roles in cancer diagnosis and prognosis assessment. Øverbye et al found that exosomal transmembrane protein 256 (TM256) in urine has high sensitivity and specificity for the diagnosis of prostate cancer. ${ }^{26}$ Exo-linc-fam $138 \mathrm{~b}$ secreted by cancer cells can inhibit the occurrence and development of liver cancer by targeting miR-765, providing new prospects for the further understanding of the complex signaling regulation of liver cancer. $^{27}$

Recent studies have shown that EphA2 can promote the growth and invasion of a variety of cancer cells. ${ }^{28-30}$ This membrane protein has a similar expression level in pure exosomes and serum. We have previously shown the diagnostic value of Exo-EphA2 combined with CA 19-9 and CA 242 in PC, but to the best of our knowledge, the effect

Table 2 Univariate and Multivariate Analysis of Overall Survival in Pancreatic Cancer

\begin{tabular}{|c|c|c|c|c|c|c|}
\hline \multirow[b]{2}{*}{ Parameters } & \multicolumn{3}{|c|}{ Univariate Analysis } & \multicolumn{3}{|c|}{ Multivariate Analysis } \\
\hline & HR & $95 \% \mathrm{Cl}$ & $P$ & HR & $95 \% \mathrm{Cl}$ & $\mathbf{P}$ \\
\hline Age & 0.99 & $0.96-1.01$ & 0.39 & & & \\
\hline Gender & 1.07 & $0.67-|.7|$ & 0.76 & & & \\
\hline Smoke & 1.11 & $0.71-1.73$ & 0.63 & & & \\
\hline Drink & 0.70 & $0.4 I-1.20$ & 0.19 & & & \\
\hline Hypertension & 1.55 & $0.88-2.72$ & 0.13 & & & \\
\hline Lymph-node-metastasis & 3.78 & $2.28-6.27$ & 0.03 & 0.99 & $0.56-1.78$ & 0.99 \\
\hline TNM stage & 5.49 & $3.29-9.16$ & 0.01 & 1.06 & $0.57-1.98$ & 0.84 \\
\hline Exo-EphA2 expression & 1.05 & $0.96-1.21$ & 0.02 & 1.04 & $1.00-1.09$ & $<0.001$ \\
\hline
\end{tabular}

Note: $P<0.05$ in bold is statistically significant.

Abbreviations: $\mathrm{Cl}$, confidence interval; $\mathrm{HR}$, hazard ratio. 
A

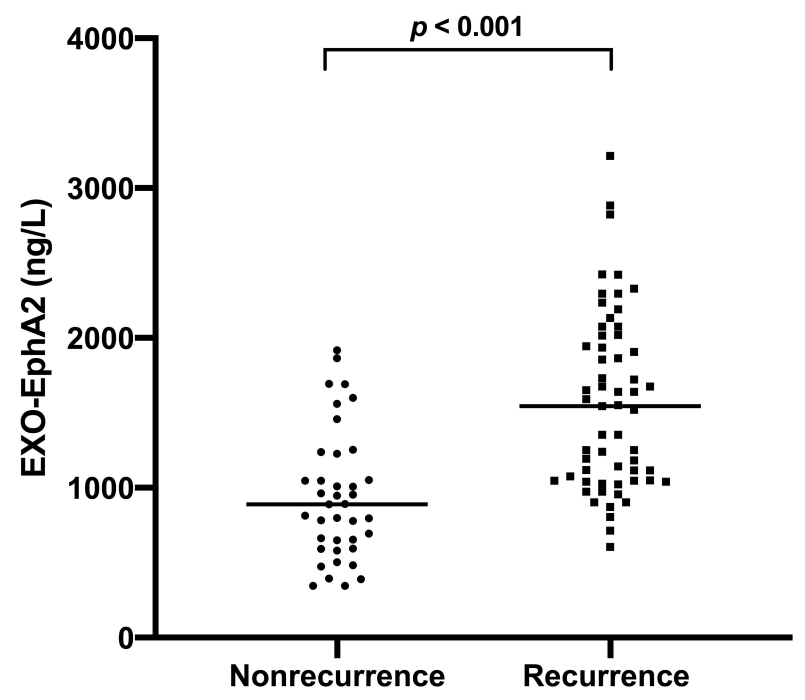

B

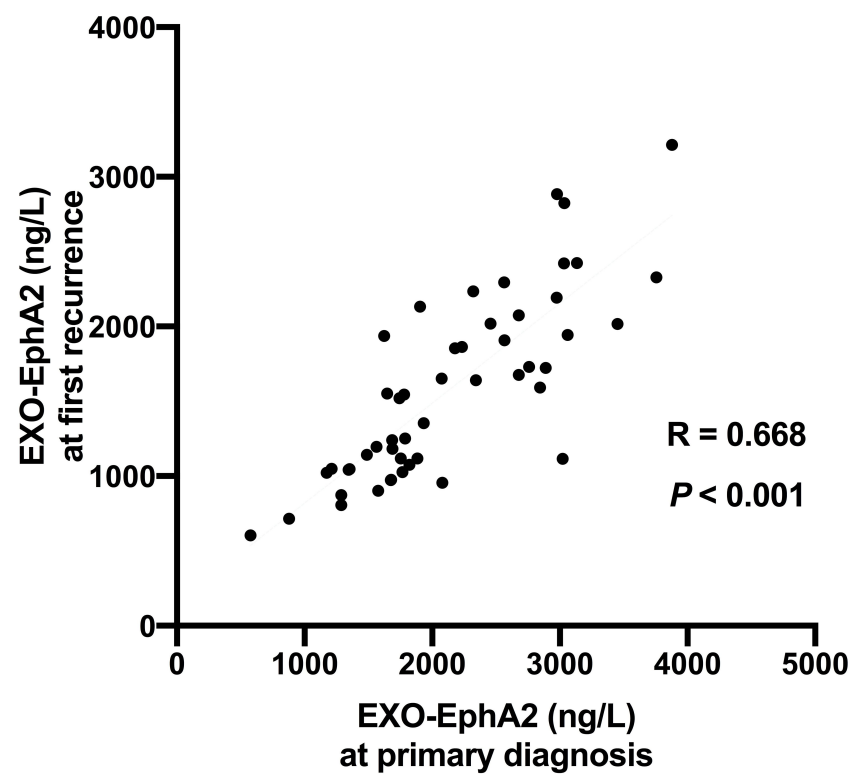

Figure 4 Serum Exo-EphA2 is upregulated in recurrent PC patients. (A) Expression of serum Exo-EphA2 in recurrent patients and non-recurrent patients ( $p<0.001)$. (B) Correlations of serum Exo-EphA2 expression at primary diagnosis and at recurrence $(p<0.00 \mathrm{I})$.

of Exo-EphA2 on the prognosis of PC remains unclear. In this study, we found that serum Exo-EphA2 was highly elevated in PC patients, and positively correlated with the tumor stage of these patients. Consistent with our previous study, which has found elevated expression of Exo-EphA2 in metastatic PC, the level of Exo-EphA2 in patients with an advanced stage disease was higher than that of patients with early stage cancer, providing an important signal for poor prognosis. Indeed, Kaplan-Meier curves of OS suggested that high expression of serum Exo-EphA2 was associated with a low OS rate. As an individual biomarker, univariate and multivariate analyses showed that the expression of serum Exo-EphA2 had a prognostic value in PC patients.
High serum Exo-EphA2 levels at the initial diagnosis indicated disease progression and poor OS. Tumor recurrences hinder outcomes, which complicates the choice of treatments. Based on this, the advantage of our study is that we analyzed serum Exo-EphA2 at both the initial diagnosis and at recurrence. We found that the expression of serum Exo-EphA2 at diagnosis in recurrent PC patients was significantly higher than that of non-recurrent PC patients. In addition, a positive correlation was found between Exo-EphA2 levels at the initial diagnosis and at recurrence. Indeed, a high Exo-EphA2 expression resulted in a poor RFS, suggesting that Exo-EphA2 could be used to guide treatment selection. However, due to the small number of patients with advanced stage, the expression of
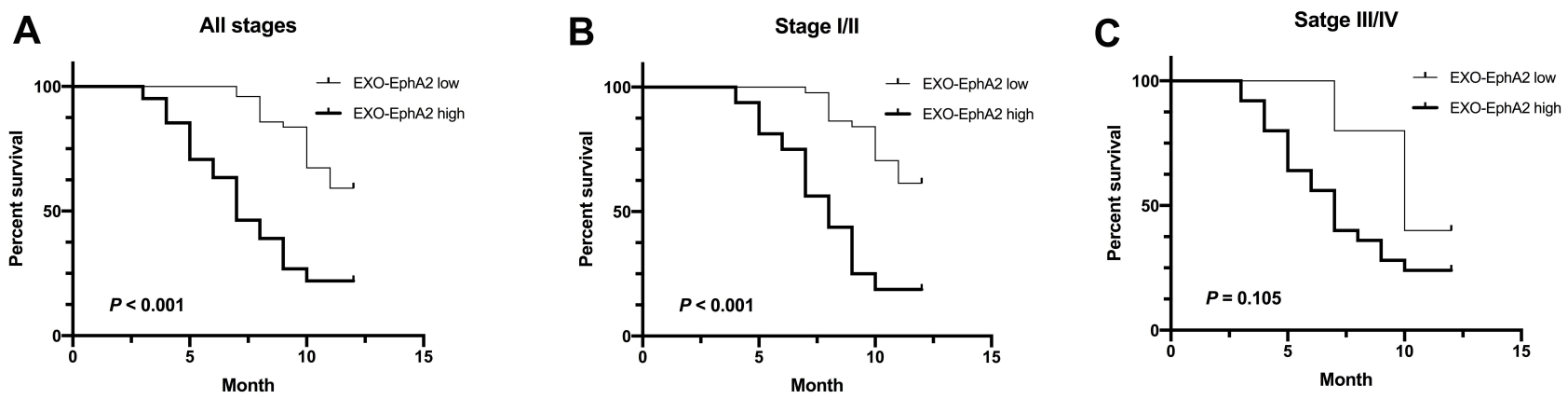

Figure 5 Higher Exo-EphA2 expression was significantly associated with poor recurrence-free survival (RFS). Kaplan-Meier curves of RFS (A) in pancreatic cancer ( $p<$ $0.00 I)$, (B) in stage I/II $(p<0.00 I)$ and $(C)$ in stage III/IV $(p=0.105)$. Based on the median level of Exo-EphA2 expression, recurrent PC patients were divided into two groups (EphA2-high expression and EphA2-low expression). 
Table 3 Univariate and Multivariate Analysis of Recurrence-Free Survival in Pancreatic Cancer

\begin{tabular}{|c|c|c|c|c|c|c|}
\hline \multirow[b]{2}{*}{ Parameters } & \multicolumn{3}{|c|}{ Univariate Analysis } & \multicolumn{3}{|c|}{ Multivariate Analysis } \\
\hline & HR & $95 \% \mathrm{Cl}$ & $P$ & HR & $95 \% \mathrm{Cl}$ & $P$ \\
\hline Age & 1.01 & $0.98-1.04$ & 0.53 & & & \\
\hline Gender & 0.76 & $0.44-1.33$ & 0.34 & & & \\
\hline Smoke & 0.83 & $0.48-1.46$ & 0.53 & & & \\
\hline Drink & 1.07 & $0.57-1.99$ & 0.83 & & & \\
\hline Hypertension & 1.14 & $0.55-2.33$ & 0.72 & & & \\
\hline Lymph-node-metastasis & 3.50 & $1.75-6.98$ & 0.03 & 1.14 & $0.5 I-2.52$ & 0.75 \\
\hline TNM stage & 2.66 & $1.55-4.59$ & 0.01 & 0.65 & $0.34-1.24$ & 0.19 \\
\hline Exo-EphA2 expression & 1.09 & $1.02-1.19$ & 0.01 & 1.41 & I.10-I.70 & 0.001 \\
\hline
\end{tabular}

Note: $P<0.05$ in bold is statistically significant.

Abbreviations: $\mathrm{Cl}$, confidence interval; $\mathrm{HR}$, hazard ratio.

Exo-EphA2 was not found to be associated with RFS in PC patients with advanced stage, which is inconsistent with our hypothesis. Univariate and multivariate analyses reiterated that the expression of serum Exo-EphA2 as an individual biomarker has a good prognostic value for PC patients with recurrence. In our previous study, we found that Exo-EphA2 could transfer chemoresistance in pancreatic cancer cells, which supports the hypothesis that a high expression of Exo-EphA2 at recurrence may be associated with chemoresistance. The selection pressure of chemotherapy triggers chemoresistance, leading to the growth of chemotherapy-resistant tumor cells. Therefore, Exo-EphA2 can be used as a blood biomarker for the therapy of $\mathrm{PC}$, predicting an invasive disease with a high recurrence risk and poor prognosis.

We acknowledge several limitations in our current research. First, this is a retrospective observational study, which may suffer from sampling biases; therefore, the conclusions need to be confirmed in a large cohort. For example, in our study, we did not find a significant correlation between smoking and poor outcome of PC patients, however, previous studies with larger sample sizes have reported that smoking was associated with reduced survival in PC patients. ${ }^{31}$ In addition, we speculated that the high levels of Exo-EphA2 might lead to chemoresistance in PC patients, however, further studies are required to investigate the clinical application value of Exo-EphA2 for therapy monitoring.

In conclusion, we provided evidence that serum ExoEphA2 is upregulated in PC. A high expression of ExoEphA2 may serve as an independent risk factor for poor OS and RFS in PC patients treated with gemcitabine-based chemotherapy. Our findings suggest that serum Exo-
EphA2 can be used as a prognostic biomarker in pancreatic cancer patients.

\section{Data Sharing Statement}

All data generated or analyzed during this study are included in this published article.

\section{Ethics Approval and Consent to Participate}

All procedures carried out in studies involving human participants were approved by the Research Ethics Committee of Tianjin Medical University Cancer Institute and Hospital (NO.2019112) and in accordance with the 1964 Helsinki Declaration ethical standards. Written informed consent was obtained from all patients, and the study was approved by the local Ethical Board.

\section{Funding}

The work was supported by the National Natural Science Foundation of China (No.81702996) and the National Science Foundation of Tianjin (18JCZDJC32600).

\section{Disclosure}

The authors declare no commercial or financial conflicts of interest.

\section{References}

1. Siegel RL, Miller KD, Jemal A. Cancer statistics, 2019. CA Cancer J Clin. 2019;69:7-34. doi:10.3322/caac.21551

2. Vincent A, Herman J, Schulick R, et al. Pancreatic cancer. Lancet. 2011;378:607-620. doi:10.1016/S0140-6736(10)62307-0

3. Zhao H, Wang Q, Wang X, et al. Correlation between RAB27B and p53 expression and overall survival in pancreatic cancer. Pancreas. 2016;45(2):204-210. doi:10.1097/MPA.0000000000000453 
4. Peinado H, Aleckovic M, Lavotshkin S, et al. Melanoma exosomes educate bone marrow progenitor cells toward a pro-metastatic phenotype through MET. Nat Med. 2012;18:883-891. doi:10.1038/nm.2753

5. Meldolesi J. Exosomes and ectosomes in intercellular communication. Curr Biol. 2018;28:R435-R444. doi:10.1016/j. cub.2018.01.059

6. Kahlert C, Kalluri R. Exosomes in tumor microenvironment influence cancer progression and metastasis. J Mol Med. 2013;91(4):431-437. doi:10.1007/s00109-013-1020-6

7. Costa-Silva B, Aiello NM, Ocean AJ, et al. Pancreatic cancer exosomes initiate pre-metastatic niche formation in the liver. Nat Cell Biol. 2015;17(6):816-826. doi:10.1038/ncb3169

8. Liu W, Hu J, Zhou K, et al. Serum exosomal miR-125b is a novel prognostic marker for hepatocellular carcinoma. Onco Targets Ther. 2017;10:3843-3851. doi:10.2147/OTT.S140062

9. Sun L, Liu X, Pan B, et al. Serum exosomal miR-122 as a potential diagnostic and prognostic biomarker of colorectal cancer with liver metastasis. J Cancer. 2020;11(3):630-637. doi:10.7150/jca.33022

10. Bryant RJ, Pawlowski T, Catto JW, et al. Changes in circulating microRNA levels associated with prostate cancer. $\mathrm{Br} J$ Cancer. 2012;106(4):768-774. doi:10.1038/bjc.2011.595

11. Miao B, Ji Z, Tan L, et al. EPHA2 is a mediator of vemurafenib resistance and a novel therapeutic target in melanoma. Cancer Discov. 2015;5:274-287. doi:10.1158/2159-8290.CD-14-0295

12. Okuyama T, Sakamoto R, Kumagai K, et al. EPHA2 antisense RNA modulates EPHA2 mRNA levels in basal-like/triple-negative breast cancer cells. Biochimie. 2020;179:169-180. doi:10.1016/j. biochi.2020.10.002

13. Lu C, Shahzad MM, Wang H, et al. EphA2 overexpression promotes ovarian cancer growth. Cancer Biol Ther. 2008;7:1098-1103. doi:10.4161/cbt.7.7.6168

14. Fan J, Wei Q, Koay EJ, et al. Chemoresistance transmission via exosome-mediated EphA2 transfer in pancreatic cancer. Theranostics. 2018;8:5986-5994. doi:10.7150/thno.26650

15. Wei Q, Wei LJ, Zhang JY, et al. EphA2-enriched exosomes promote cell migration and are a potential diagnostic serum marker in pancreatic cancer. Mol Med Rep. 2020;22(4):2941-2947. doi:10.3892/ mmr.2020.11384

16. Wei Q, Zhang J, Li Z, et al. Serum Exo-EphA2 as a potential diagnostic biomarker for pancreatic cancer. Pancreas. 2020;49 (9):1213-1219. doi:10.1097/MPA.0000000000001660

17. Fu Z, Jiao Y, Li Y, et al. TYMS presents a novel biomarker for diagnosis and prognosis in patients with pancreatic cancer. Medicine. 2019;98(51):e18487. doi:10.1097/MD.0000000000018487

18. Drouillard A, Puleo F, Bachet JB, et al. DLL4 expression is a prognostic marker and may predict gemcitabine benefit in resected pancreatic cancer. $B r \quad J$ Cancer. 2016;115(10):1245-1252. doi:10.1038/bjc.2016.319
19. Deng GC, Yan H, Guo ZP, et al. Correlation between baseline serum tumor markers and clinical characteristic factors in patients with advanced pancreatic cancer. Onco Targets Ther. 2020;13:11151-11163. doi:10.2147/OTT.S269720

20. Ma W, Li T, Wu S, et al. LOX and ACSL5 as potential relapse markers for pancreatic cancer patients. Cancer Biol Ther. 2019;20 (6):787-798. doi:10.1080/15384047.2018.1564565

21. Steinbichler TB, Dudás J, Riechelmann H, et al. The role of exosomes in cancer metastasis. Semin Cancer Biol. 2017;44:170-181. doi:10.1016/j.semcancer.2017.02.006

22. Becker A, Thakur BK, Weiss JM, et al. Extracellular vesicles in cancer: cell-to-cell mediators of metastasis. Cancer Cell. 2016;30 (6):836-848. doi:10.1016/j.ccell.2016.10.009

23. Mashouri L, Yousefi H, Aref AR, et al. Exosomes: composition, biogenesis, and mechanisms in cancer metastasis and drug resistance. Mol Cancer. 2019;18(1):75. doi:10.1186/s12943-0190991-5

24. Zou SL, Chen YL, Ge ZZ, et al. Downregulation of serum exosomal miR-150-5p is associated with poor prognosis in patients with colorectal cancer. Cancer Biomark. 2019;26(1):69-77. doi:10.3233/CBM190156

25. Lv S, Wang $\mathrm{Y}, \mathrm{Xu} \mathrm{W}$, et al. Serum exosomal miR-17-5p as a promising biomarker diagnostic biomarker for breast cancer. Clin Lab. 2020;66(9). doi:10.7754/Clin.Lab.2020.200127.

26. Øverbye A, Skotland T, Koehler CJ, et al. Identification of prostate cancer biomarkers in urinary exosomes. Oncotarget. 2015;6 (30):30357-30376. doi:10.18632/oncotarget.4851

27. Zhuo C, Yi T, Pu J, et al. Exosomal linc-FAM138B from cancer cells alleviates hepatocellular carcinoma progression via regulating miR-765. Aging. 2020;12(24):26236-26247. doi:10.18632/ aging. 202430

28. London M, Gallo E. The EphA2 and cancer connection: potential for immune-based interventions. Mol Biol Rep. 2020;47(10):8037-8048. doi:10.1007/s11033-020-05767-y

29. Huang C, Yuan W, Lai C, et al. EphA2-to-YAP pathway drives gastric cancer growth and therapy resistance. Int $J$ Cancer. 2020;146(7):1937-1949. doi:10.1002/ijc.32609

30. Kinch MS, Carles-Kinch K. Overexpression and functional alterations of the EphA2 tyrosine kinase in cancer. Clin Exp Metastasis. 2003;20:59-68. doi:10.1023/A:1022546620495

31. Yadav D, Lowenfels AB. The epidemiology of pancreatitis and pancreatic cancer. Gastroenterology. 2013;144(6):1252-1261. doi:10.1053/j.gastro.2013.01.068

\section{Publish your work in this journal}

Cancer Management and Research is an international, peer-reviewed open access journal focusing on cancer research and the optimal use of preventative and integrated treatment interventions to achieve improved outcomes, enhanced survival and quality of life for the cancer patient.
The manuscript management system is completely online and includes a very quick and fair peer-review system, which is all easy to use Visit http://www.dovepress.com/testimonials.php to read real quotes from published authors. 